\title{
ORAL GOLDS
}

\author{
David Brown \\ Guy's Hospital Medical and Dental Schools, London SE1 9RT
}

\begin{abstract}
The aim of what we now call restorative dentistry is to replace teeth lost by either accidental or intentional extraction, or to replace parts of teeth lost due to decay or accident. In this short review, aimed at the general reader, the author describes gold's important role in dentistry.
\end{abstract}

\section{Fillings and Castings}

Modern techniques and materials enable the dental surgeon and dental technician to create a wide range of restorations. Some materials, such as dental amalgam, cements and composites, are manipulated as pastes directly in the mouth of the patient, and set to become strong and rigid parts of the tooth's anatomy. Modern dental alloys, however, many of which are based on gold, are cast in the laboratory and then cemented into place in the mouth.

\section{The Use of Pure Gold}

The durability of gold and its inherent resistance to environmental degradation has left us with a number of examples from antiquity of dental restorations. These were often made of gold, which had been formed to produce bands capable of holding replacements from lost teeth. Such teeth were either 'borrowed' from a donor or in some cases were fabricated from the ivory tusks of the elephant, hippopotamus or walrus. As man's technological skills grew, the ivory was replaced with specially-developed porcelains, but the bases to which the porcelain teeth were attached were thin gold plates beaten to shape over models of the gums made from plaster or low-melting point alloys. This practice of swaging gold plates continued well into the 20th century. However, the only mechanical forming to which gold is subjected for dental use in the 1980s, and then very rarely, is its use in the cohesive gold technique. Typical of this procedure is the use of thin leaves of $99.99 \%$ gold, which are rolled into cylinders, annealed to both dry them and drive off any absorbed surface gases, and then condensed incrementally into small, carefullyprepared cavities in well-supported teeth, using either a hand mallet or a mechanical condenser. During this condensing the gold is both closely adapted to the surrounding tooth and cold-welded to form cohesive bonds be- tween each increment. The result is a restoration which does not leak and has great durability. However, it is a procedure which is very time-consuming and limited to simple cavities with good access, in patients with little dental decay and in sites where little wear is anticipated.

\section{Lost-wax Casting}

For restorations which are going to be subjected to both loading and abrasion during their time in a normal mouth, a resistance to deformation and wear is an obvious necessity. These restorations take the form of inlays (which replace part of the tooth), crowns (which replace most or all of the outer surface of the tooth), or bridges (which replace missing teeth). Their close fit to the teeth is obtained by precision casting techniques. However, the first stage after the teeth have been prepared is to take an impression in a material which flows in a controlled way into the areas of interest and then sets. From this negative impression a positive model is made in hard plaster, and this is used by the dental technician to produce a wax pattern of the inlay, crown or bridge. This pattern is then invested in a mixture of refractory silica and a binding agent, often gypsum. When it is set, the wax is burned away in a furnace and the appropriate dental alloy is forced by pressure into the space within the investment formerly occupied by the wax. It solidifies and can then be removed, cleaned and polished before being sent to the dental surgeon, who cements it into or onto the prepared teeth.

\section{Dental Casting Procedures}

Producing small castings of great dimensional accuracy is achieved in dentistry in two ways. Firstly, to compensate for the inevitable shrinkage, which occurs when the metal solidifies, the space formerly occuped by the wax is expanded by heating the invested pattern space. Not only 


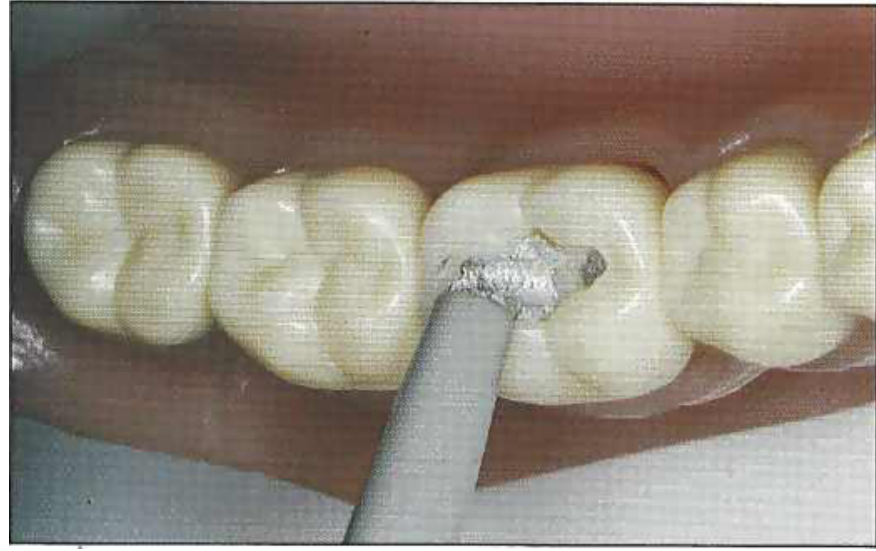

Fig. 1a Dental amalgam (silver-tin-copper and mercury) being extruded as a plastic mass into an undercut, retentive cavity

Photograph by courtesy of Mr R. Ellis, U.M.D.S, Guy's Hospital, London

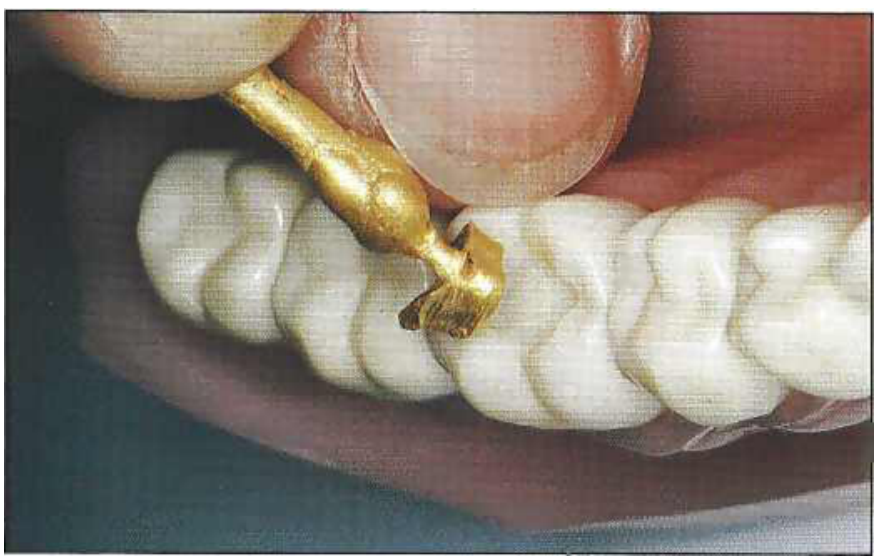

Fig. 1b A Type II dental gold inlay casting being tried on a model of the patient's prepared tooth, prior to being trimmed and polished

Photograph by courtesy of Mr R. Ellis, U.M.D.S, Guy's Hospital, London

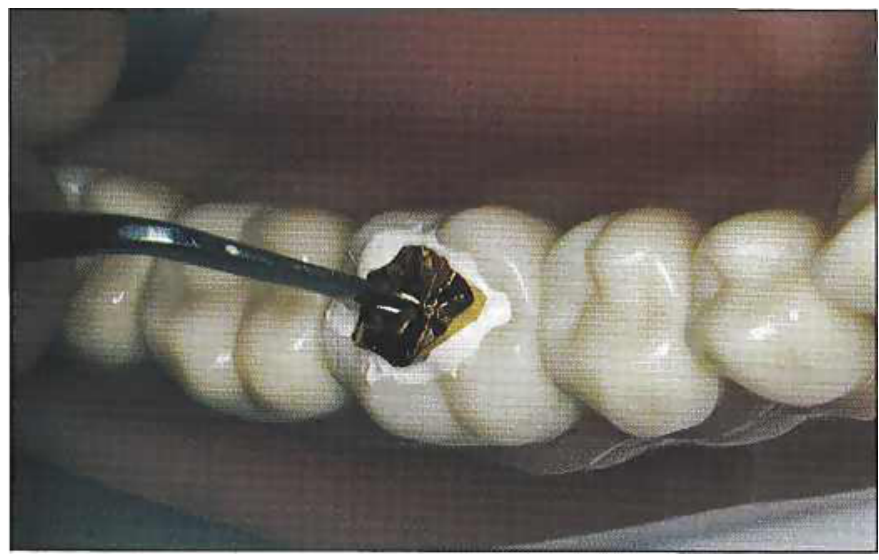

Fig. 1c The completed gold inlay being cemented into a non-undercut cavity

Photograph by courtesy of Mr R. Ellis, U.M.D.S, Guy's Hospital, London does the silica refractory in the investment undergo normal thermal expansion, but it also enjoys a structural inversion. In this the bonds between the silicon and the oxygen, which are at $90^{\circ}$ to each other at low temperatures, transform to $180^{\circ}$ at an inversion temperature which is characteristic for each of the many allotropes of silica. The other requirement is that the molten alloy should be encouraged to flow into the thinnest sections of the pattern space, and the techniques by which this is brought about have evolved together with the use of dental golds. The earliest method of producing a pressure of metal used a device rather like a censer. The invested pattern and a crucible was placed in a tray on chains, the alloy was melted, and the operator generated a centrifugal force by swinging the tray vigorously in a circle. Centrifugal casting, using a spring-loaded, cranked-arm machine, is still in use today, although the trend is now towards a machine which allows the alloy to be melted by electrical resistance heating under vacuum, and then applies a positive pressure to force the molten alloy into the mould. This use of pressure is not new, but when it was used in the past the alloy was melted in the investment cone prior to applying the pressure. The pressure has sometimes been created by air and sometimes by steam generated from damp asbestos. When not brought about in one of the modern integrated casting units, melting of the dental gold alloy has usually been by gas-air torch.

\section{Dental Gold Alloys}

What is the 'appropriate alloy' mentioned above? Ever since the lost-wax casting process has been used in dentistry the search has been on for the ultimate material - one which combines ease of manipulation, strength, stiffness, durability, resistance to surface tarnish and sub-surface corrosion, with economy.

\section{The Specification Alloys}

For more than 50 years alloys whose major constituent was gold have been used for the production of inlays, crowns and bridges. In the 1920s the alloys were classified according to their mechanical properties. They were, and still are known as Type I (A, or soft), Type II (B, or medium), Type III (C, or hard) and Type IV (D, or extrahard). These are sometimes known as the 'Specification Alloys', as they comply with national and international standards, including BS 4425:1969, which requires that they contain no less than $75 \%$ of the noble metals gold, platinum and palladium. The other $25 \%$ is usually a combination of copper, silver and zinc.

The softest material, which can be used for very small 


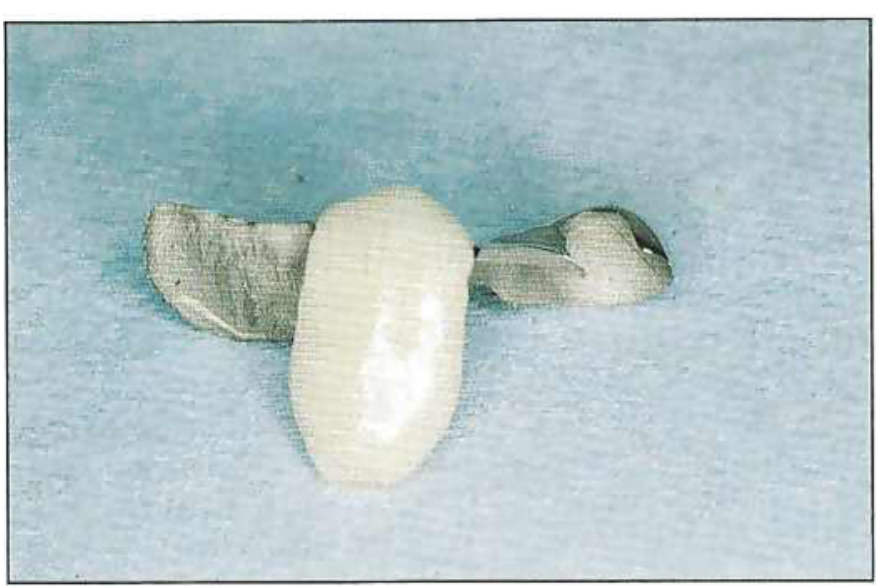

Fig. 2 A modern nickel-chromium alloy 'Maryland' bridge with a dental porcelain facing. The electrolytically-etched surface of the metal framework will be bonded to the rear, acid-etched surfaces of the adjacent teeth using a resin cement. Photograph by courtesy of Dr B.G.N. Smith, U.M.D.S, Guy's Hospital, London

dental inlays that are not going to be highly stressed, is the Type I alloy (19-22 carat: $800-900$ fine), containing about $10 \%$ silver and $5 \%$ copper. This type of alloy has a maximum hardness of 75BHN, and an elongation to failure of $25 \%$. This means that it can be readily burnished. This is a process by which the dental technician or the dentist can cause the edge of a thin casting to flow by applying pressure, usually through a rotating, smooth dental bur. It is a technique which can help in forming a good seal at the margin between the casting and the tooth when the former is cemented to the latter.

For inlays likely to be exposed to higher stress, a Type II alloy is the material of choice. This is $18-19$ carat or 750-780 fine. It contains up to $15 \%$ silver, $10 \%$ copper, $4 \%$ palladium, $1 \%$ platinum and $1 \%$ zinc, but never less than $75 \%$ noble metals. Such an alloy would show an elongation to failure of about $24 \%$ and can thus easily be burnished. However, with a hardness of $100 \mathrm{BHN}$ it is harder than the Type I alloy, but not as hard as the Type III alloy, which has a hardness of $140 \mathrm{BHN}$ when cast and 180BHN when hardened. Unlike the Types I and II alloys, the Types III and IV alloys can be heat-treated to increase their hardness. This hardening process is generally attributed to the formation of a superlattice or ordered solid solution between the gold and copper atoms. This superlattice interferes with dislocation movement, and the mechanical properties (as indicated by the hardness values) are modified accordingly.

The Type III alloys is widely used for crowns and for bridges where no porcelain facing is required. It is an alloy of 15-19 carat: $620-780$ fine, containing up to $26 \%$ silver, $11 \%$ copper, $4 \%$ palladium, $3 \%$ platinum and $1 \%$ zinc, but never less than $75 \%$ noble metals.

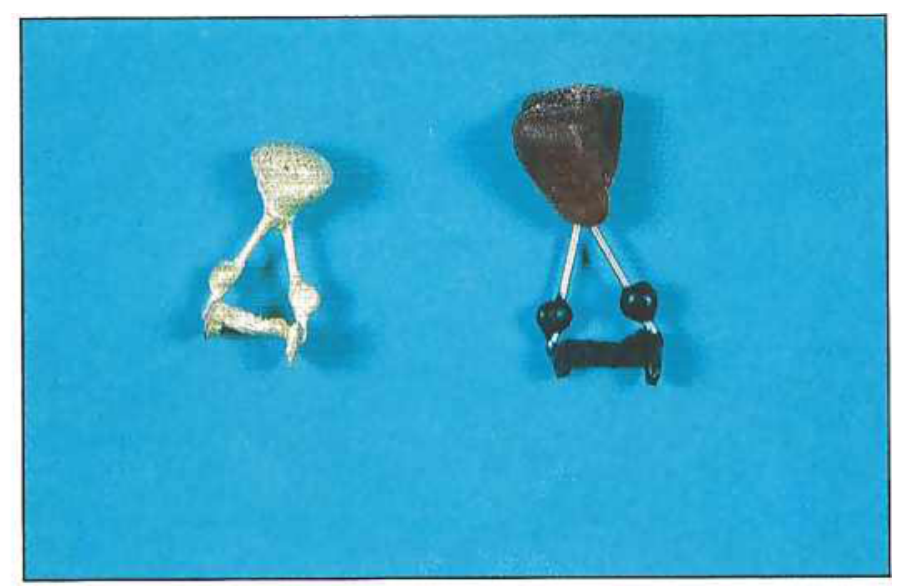

Fig. 3 A Type II dental gold inlay and a copy of the sprued wax pattern used to produce it. The patterns was invested in gypsum-bonded silica. Then this had set, the metal sprues were removed prior to heating the mould to burn out the wax and expand the mould.

The extra-hard, Type IV alloy is at the limit of the permitted composition range. It is 14-17 carat: $600-700$ fine containing up to $20 \%$ silver, $16 \%$ copper, $5 \%$ palladium, $4 \%$ platinum and $2 \%$ zinc, but never less than $75 \%$ noble metals. As cast it has a Brinell hardness of 150 , but after heat treatment first at $700^{\circ} \mathrm{C}$ followed by another at $420^{\circ} \mathrm{C}$ for 10 minutes, raises this to $230 \mathrm{BHN}$. This alloy was used in the past for casting denture frameworks, but nowadays only clasps of partial dentures are made from this alloy. The clasps can be bent into shape with the alloy in the heatsoftened condition and then hardened for use. Neither the Type III or Type IV alloys can be burnished, as their elon-

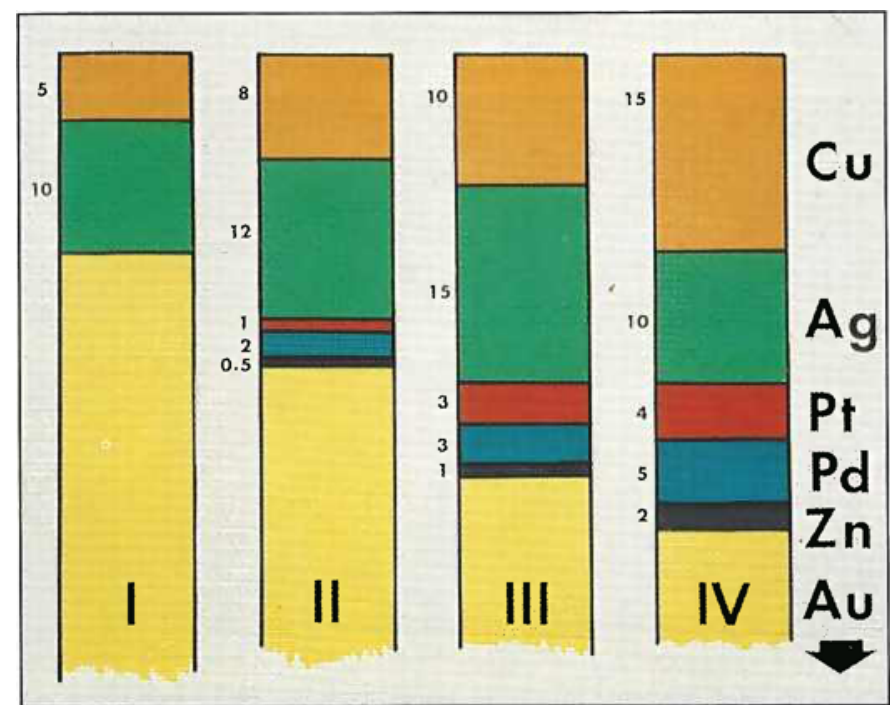

Fig. 4 Diagram demonstrating the composition differences of some typical high-gold specification alloys, Types I-IV 
gation to failure is down to no more than $10 \%$ when hardened.

These then are the original, high gold 'Specification Alloys', on which the dental profession has built its reputation over the last 70 years, and they provide the yardstick for the imitators, which now seek to displace them. Economic forces are now pushing hard at the door and unfortunately the profession world-wide is being tempted to use inferior alloys.

\section{Medium- and Low-gold Alloys}

Whilst it is relatively easy to reproduce the hardness values of the high-gold alloys in other systems, this is invariably at the expense of some of the other properties. In particular, the medium- and low-gold alloys, which are now available to the profession, can develop an inferior tarnish resistance during handling, and this can produce distressing results for the patient.

In attempts to control the proliferation of inferior alloys, national and international standards have been modified. For example, in the United Kingdom these new alloys are required to comply with BS6042:1981. This specification describes these medium-gold alloys as semi-precious, and requires that they contain not less than $30 \%$ of the noble metals gold, platinum and palladium, and not more than $20 \%$ of base metals such as copper and zinc. The remainder is silver, and in practice they are generally silver-palladium-gold-copper alloys, which have a similar range of mechanical properties to the high-gold alloys.

To add to the confusion, the American Dental Associa-

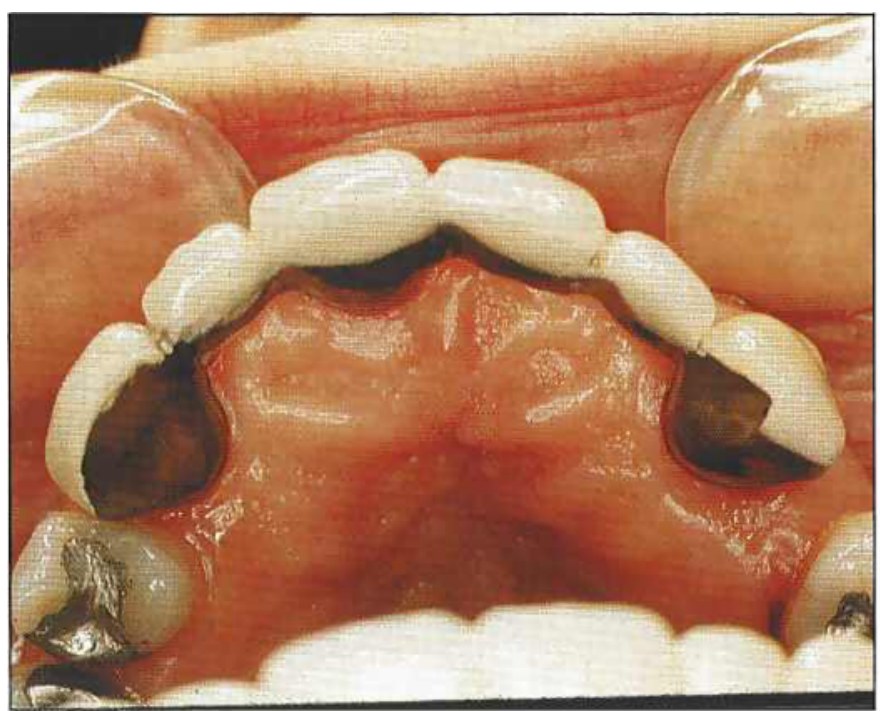

Fig. 5 A six-unit bridge made from platinised-gold and faced with dental porcelain. The teeth at either end provide the support for the four central teeth. Two amalgam fillings are also visible.

Photograph by courtesy of Dr B.G.N. Smith, U.M.D.S, Guy's Hospital, London tion (ADA) now has an alternative classification for dental alloys, which is intended to help dentists and technicians in selecting suitable materials. In this classification, those alloys which contain at least $60 \%$ of the noble metals and at least $40 \%$ of gold are called 'High Noble'. Those which contain at least $25 \%$ gold, platinum or palladium are called 'Noble', and those which contain less than $25 \%$ of these noble metals are classified as 'Predominantly Base'. The ADA 'Noble' alloys are really silver-palladium alloys, and when these were in use in dentistry in the 1960s they were called the 'white golds' . They contained $30-45 \%$ silver, $20-25 \%$ palladium, $15-30 \%$ gold, 15-20\% copper and 1\% zinc. Modern 'white gold' alloys may contain as little as $2 \%$ gold, together with base metals such as tin and indium. In general they have a hardness of about 170BHN and are prone to brittleness if slowly cooled. They also tend to work harden rapidly, which precludes excessive adjustment or any burnishing. Tarnishing in the mouth is common with these alloys.

\section{Resin-bonded Bridges}

An application in which gold alloys are now tending to be ousted is a new dental technique, in which a single missing tooth is replaced by a porcelain-faced metal framework, which is bonded to the rear of the adjacent teeth without having to damage them. Prior to the development of this design of 'resin-bonded bridge', it was necessary to prepare the teeth on either side of the missing tooth. This involved the cutting away of sound enamel and den-

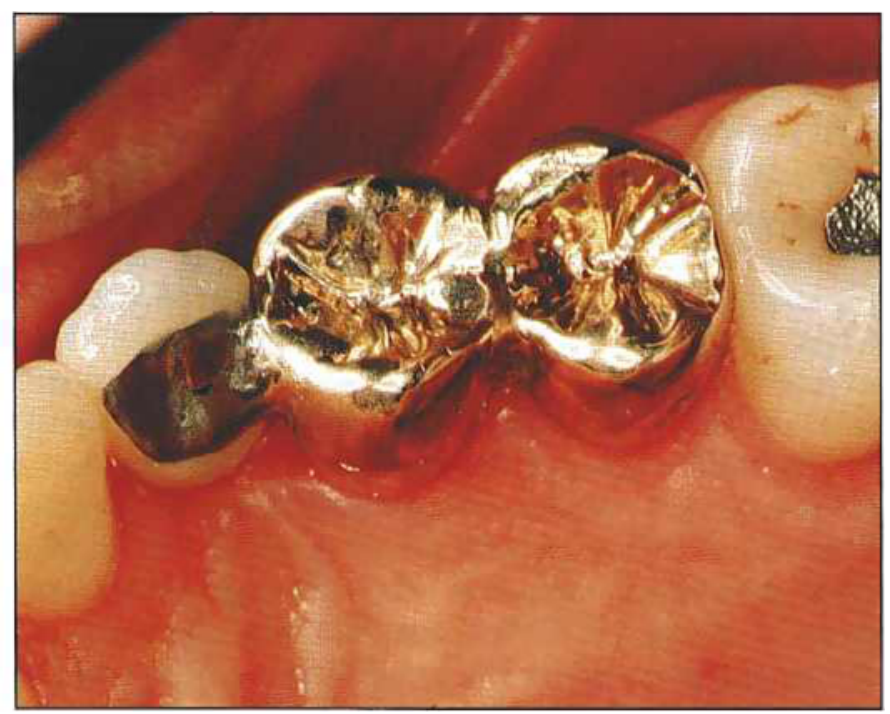

Fig. 6 A two-unit, Type III dental gold alloy bridge soldered to a platinised gold crown with a dental porcelain facing

Photograph by courtesy of Dr B.G.N.Smith, U.M.D.S, Guy's Hospital, London 


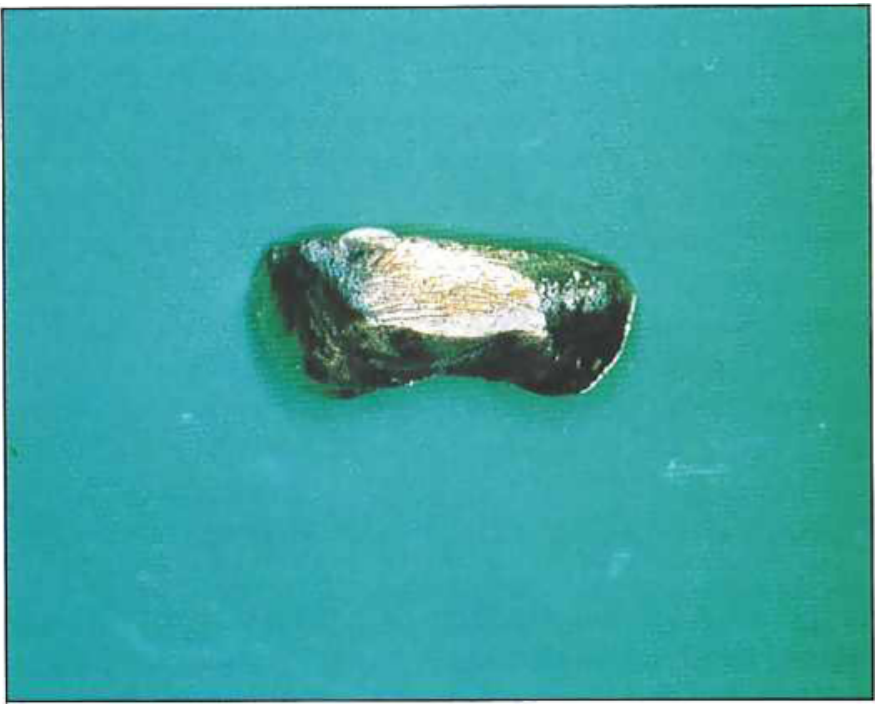

Fig. 7 Part of a crown made from a medium-gold alloy (to BS6042:1981) after being removed from a patient's mouth because of severe tarnishing. Analysis showed the base metal content of this crown to be 23.2\%, probably due to contamination of the alloy during casting.

tine. Once this had been done, an impression was taken and a model was made. A wax pattern was produced, invested and cast, such that between two metal thimbles called abutments there was a bridge or pontic. To this metal bridge porcelain was added and fired to simulate the missing tooth and the adjacent teeth, which had been damaged in the preparation of the abutments. The alloys for this application need to possess special properties. For example, a platinised, high-gold alloy for this purpose would typically contain up to $6 \%$ platinum, $5 \%$ palladium, $1 \%$ silver and traces of indium, iron and tin. These latter elements are added to assist in forming a chemical bond with the porcelain. Copper, which turns the porcelain green, is absent. These alloys require special casting techniques and investment materials, and are very expensive.

A number of high-modulus, base-metal alloys to which porcelain is to be fired had been in development since the 1960 s as alternatives to platinised-gold. Some of these were based on nickel-chromium, and others on cobaltchromium. Researchers in the University of Maryland discovered that it was possible electrolytically to etch some of these alloys to produce a pitted surface. It was also relatively easy to produce etch pits in sound dental enamel using phosphoric acid, and with resin-based dental cements it proved feasible to permanently bond the two etched surfaces. Hence, by modifying the design of the bridge and by merely etching the rear surfaces of the teeth adjacent to the gap, it was possible to replace a single tooth with an absolute minimum of damage to the neighbouring teeth. Such restorations rely on the inherently high

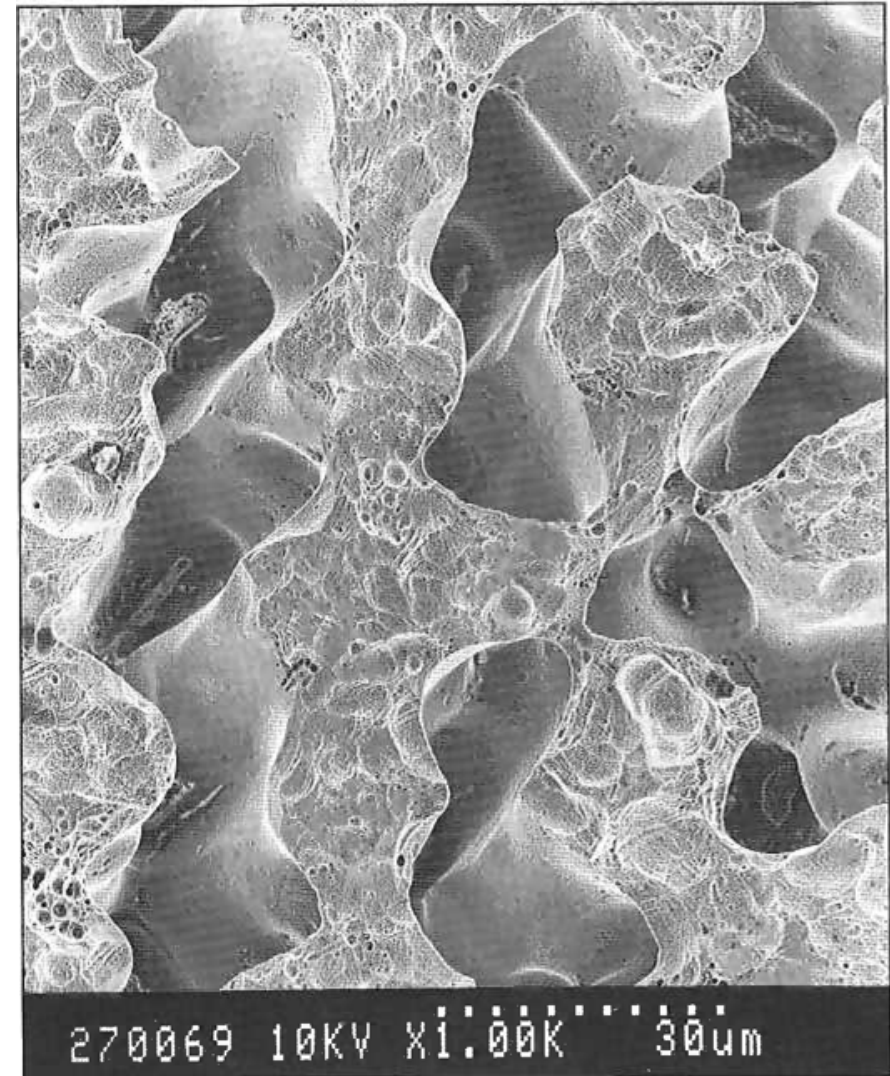

Fig. 8 A scanning electron micrograph of the electrlytically-etched surface of a nickel-chromium alloy intended for use in the construction of a Maryland-type of bridge. These bridges are bonded to acid-etched enamel via a resin-based cement, which flows into the etch pits in both surfaces and sets. Photograph courtesy of Mr O. Atta, U.M.D.S., Guy's Hospital, London

modulus of the base-metal alloys. This enables the metal 'wings', which need to be made in thin section, to resist elastic deformation during normal use.

A considerable number of these alloys have now been developed, but, as yet, none of them is a gold-based one. However, materials scientists are continuing their search, for as we have known for over 1000 years, there is no real substitute for gold in the oral cavity.

\section{Further reading}

1 J.A. Donaldson, 'The Use of Gold in Dentistry', Gold Bull., 1980, 13(3), 117-124 and 1980, 13, (4), 160-165

2 H. Knosp, M. Nawaz and M. Stumke, 'Dental Gold Alloys', Gold Bull., 1981, 14(2), 57-64

3 L.W. Laub and J.W. Standford, 'Tarnish and Corrosion Behaviour of Dental Gold Alloys', Gold Bull., 1981, 14(1), 13-18

4 L.B Hunt, 'The Long History of Lost Wax Casting', Gold Bull., 1980, 13(2), 63-79 